\title{
Le gouvernement du Canada doit continuer à jouer de prudence dans le dossier de l'aide médicale à mourir
}

\author{
Andreas Laupacis MD MSc \\ Citation: CMAJ 2020 February 24;192:E188-9. doi: 10.1503/cmaj.200213-f \\ Voir la recherche connexe (en anglais) à www.cmaj.ca/lookup/doi/10.1503/cmaj.200016
}

$\mathbf{L}$ a légalisation de l'aide médicale à mourir en juin 2016 est le changement clinique le plus profond que j'ai connu dans ma carrière de médecin. Rien n'est plus marquant et émotif qu'un décès; or, en l'espace d'une journée, des Canadiens ont littéralement obtenu la possibilité de choisir entre vivre et mourir.

La légalisation nous a imposé de faire primer les volontés des patients par-dessus tout, mais nous devons en même temps respecter le point de vue de chaque professionnel sur l'aide à mourir et veiller à ce que seules les personnes satisfaisant les critères énoncés dans la loi fédérale se la voient offrir. Pour recevoir l'aide médicale à mourir au Canada, il faut être lucide au moment de l'intervention, avoir une maladie qui causera la mort de façon raisonnablement prévisible et connaître des souffrances physiques ou psychologiques intolérables malgré l'accès à des traitements.

Actuellement, le gouvernement du Canada examine la possibilité d'élargir ces critères. L'analyse des 2241 premiers cas d'aide médicale à mourir en Ontario par Downar et ses collaborateurs arrive donc à point ${ }^{1}$. Les chercheurs ont relevé que par rapport à l'ensemble des personnes décédées dans la province, celles qui avaient reçu l'aide médicale à mourir étaient proportionnellement plus nombreuses à avoir un diagnostic de cancer, à être mariées et à vivre dans un quartier nanti. Les trois quarts d'entre elles recevaient des soins palliatifs avant leur décès. La procédure a eu lieu dans les 10 jours de réflexion prévus pour $58 \%$ des gens dont il restait moins d'un mois à vivre. Et la famille ou les professionnels se sont inquiétés de difficultés d'accès dans $7 \%$ des cas d'intervention.

Ces résultats sont somme toute rassurants. Il est important de recueillir et de divulguer publiquement des données sur toutes les personnes qui reçoivent l'aide médicale à mourir au pays. Aussi les autres provinces devraient-elles publier le même genre de recherches.

Les constats des auteurs devraient dissiper cette crainte voulant que les Ontariennes et Ontariens qui cherchent à obtenir l'aide médicale à mourir font ce choix parce qu'ils ont un faible soutien social ou un accès restreint aux soins. En fait, l'étude prouve le contraire. Il faudrait donc, à l'inverse, veiller scrupuleusement à ce que les personnes admissibles qui sont socialement ou économiquement vulnérables sachent qu'elles ont droit à l'aide à mourir.

C'est encourageant de voir que pour $58 \%$ des malades ayant un pronostic vital de moins d'un mois, le médecin a voulu et a pu procéder avant la fin de la période de réflexion de 10 jours prévue par la loi, permettant au patient de mourir comme il le souhaitait. Peut-être faudrait-il laisser tomber ce délai quand la mort naturelle est imminente.... Une question que la fréquence d'abandon des démarches pendant ces 10 jours, si elle était connue, nous aiderait à trancher.

Bien qu'elle révèle que peu de gens s'inquiètent de possibles difficultés d'accès, l'étude est muette quant au nombre de patients dont l'accès à la procédure est si difficile qu'il en devient impossible. Voilà un sujet à creuser. Elle ne traite pas non plus de l'aide médicale à mourir dans les établissements confessionnels, où les patients devraient pouvoir l'obtenir aussi rapidement qu'ailleurs.

Au moment de la légalisation, Ottawa a adopté une approche de «prudence, mesure et évaluation ». C'était le bon choix : la première évaluation est de bon augure, du moins pour l'Ontario.

Il semble que l'aide médicale à mourir au Canada entre dans une nouvelle ère. Dernièrement, la Cour supérieure du Québec a statué que le refus de la pratiquer sur deux requérants - qui sont lourdement handicapés - seulement parce qu'ils ne répondent pas au critère de mort raisonnablement prévisible, était inconstitutionnel ${ }^{2}$. Dans la foulée, le gouvernement fédéral s'est engagé à assouplir les critères d'admissibilité pour ne plus réserver l'accès aux personnes en fin de vie ${ }^{3}$. De plus, il examine la possibilité de légaliser l'aide à mourir pour 3 autres groupes : les mineurs matures, les personnes ayant la maladie mentale comme seul motif, et les personnes qui veulent faire une demande anticipée en prévision 
d'une incapacité à formuler leur requête plus tard (en raison d'une démence profonde, par exemple).

Il y a plus d'un an, le Conseil des académies canadiennes a publié un rapport exhaustif et éclairé sur chacune de ces 3 situations ${ }^{4}$. Même s'il y a peu de précédents à l'international sur lesquels se baser, la vulnérabilité particulière des groupes susmentionnés soulève des préoccupations éthiques importantes auxquelles il faut clairement répondre.

Autoriser l'aide médicale à mourir pour les adolescentes et adolescents de 17 ans jugés matures dont les souffrances dues à un cancer avancé sont inapaisables ne nécessiterait que des changements relativement mineurs aux mesures de protection en place. L'autoriser pour les personnes isolées socialement qui ont une maladie chronique avancée ou qui sont lourdement handicapées et dont la mort n'est pas prévisible, c'est autre chose. Celles-ci ayant parfois de la difficulté à obtenir des soins de grande qualité, il faut veiller à ce qu'elles en reçoivent avant même de songer à leur offrir l'aide à mourir.

Si les critères d'admissibilité venaient à être élargis, il faudrait implanter des mesures de protection propres à chaque nouvelle indication. Et enfin, continuer à jouer de prudence, à mesurer méticuleusement les résultats et à réévaluer la situation.

\section{Références}

1. Downar J, Fowler RA, Falko R, et al. Early experience with medical assistance in dying in Ontario, Canada: a cohort study. CMAJ 2020;192:E173-81.

2. Jessome J. Canada: Truchon v. Procureur général du Canada: Superior Court of Quebec finds limiting access to medical assistance in dying ("MAiD") to end of life unconstitutional. mondaq; 2019. Accessible ici : www.mondaq.com/canada /Food-Drugs-Healthcare-Life-Sciences/855424/Truchon-v-Procureur-Gnral-Du -Canada-Superior-Court-Of-Quebec-Finds-Limiting-Access-To-Medical-Assistance -In-Dying-Maid-To-End-Of-Life-Unconstitutional (consulté le 6 février 2020).

3. Ministère de la Justice Canada. Le gouvernement du Canada consulte les Canadiens sur l'aide médicale à mourir [communiqué de presse]. Ottawa : Ministère de la Justice Canada; 2020. Accessible ici : www.canada.ca/fr/ministere-justice/nouvelles /2020/01/le-gouvernement-du-canada-consulte-les-canadiens-sur-laide-medicale -a-mourir.html (consulté le 6 février 2020).

4. Comité d'experts sur l'aide médicale à mourir. L'état des connaissances sur l'aide médicale à mourir pour les mineurs matures, les demandes anticipées et les demandes lorsqu'un trouble mental est le seul problème médical invoqué : sommaire des rapports. Ottawa : Conseil des académies canadiennes; 2018. Accessible ici : https://rapports-cac.ca/wp-content/uploads/2019/02/Sommaire -des-rapports.pdf (consulté le 6 février 2020).

Intérêts concurrents : Se rendre à www.cmaj.ca/site/misc/cmaj staff.xhtml

Affiliation : Rédacteur en chef, CMAJ

Correspondance à : Éditeur du CMAJ, cmaj@cmajgroup.ca 